\title{
Interpersonal Cognitive Biases in Children and Young People with Neurodevelopmental Disorders: A Systematic Review
}

\author{
Nora B. Schmidt ${ }^{1}$. Leen Vereenooghe ${ }^{1}$ \\ Accepted: 20 October 2021 / Published online: 17 November 2021 \\ (c) The Author(s) 2021
}

\begin{abstract}
Purpose of Review Interpersonal cognitive biases have been linked to externalising and internalising problems. This systematic review investigates their role in children and young people with neurodevelopmental disorders (NDD), who have a high risk of experiencing such problems.

Recent Findings With 16 identified studies, this is a widely under-recognised research area. The three studies conducted within the last 5 years focused on threat interpretation and its association with anxiety. No difference between children and young people with and without NDD was found in the eleven studies investigating hostile attribution of intent, of which the most recent is nearly a decade old. No studies addressed attention or memory bias towards ambiguous interpersonal information.

Summary The scarcity and heterogeneity of research highlighted in this paper demonstrate the urgency to use standardised and accessible research methods to develop a strong evidence base regarding the potential content-specific interpretation bias in individuals with NDD.
\end{abstract}

Keywords Neurodevelopmental disorders · Cognitive bias · Interpretation bias · Hostile attribution · Causal attribution . Systematic review

\section{Introduction}

Different neurodevelopmental disorders (NDD), which include intellectual disability (ID), autism spectrum disorder (ASD), attention-deficit/ hyperactivity disorder (ADHD), specific learning disorders (SLD), and motor and communication disorders, frequently co-occur and show overlap in their psychiatric comorbidities with both externalising and internalising problems and in their cognitive and social cognitive deficits [1-9]. Aggressive behaviour problems, which are frequently higher in children and young people (CYP) with NDD than in typically developing (TD) controls [10-13], are increasingly approached in individuals with NDD using the social information processing (SIP) model, most commonly in individuals with ID $[14,15,16 \bullet]$.

Leen Vereenooghe

leen.vereenooghe@uni-bielefeld.de

1 Faculty of Psychology and Sports Science, Bielefeld University, Bielefeld, Germany
The SIP model views aggression as the result of a tendency to show biased or deficient encoding and interpretation of interpersonal cues, to select antisocial rather than prosocial goals and to generate, positively evaluate, select, and enact incompetent rather than competent behavioural responses $[17,18 \bullet, 19,20]$.

People may show attention bias or memory bias at the encoding stage of SIP if they selectively attend or recall negative social cues when being asked to imagine a hypothetical scenario in which the protagonist experiences an undesirable outcome as a result of another person's actions [21-24]. The tendency to attribute hostile intent to the other person, when that person's actual intent is ambiguous or non-hostile, points to a bias at the interpretation stage referred to as hostile attribution of intent (HAI) [25].

Attention, memory, and interpretation biases may play a role in causing or maintaining externalising and internalising problems [26-28]. For instance, individuals high in aggression are characterised by HAI [29•] and people high in social anxiety show social threat interpretation bias, such as interpreting the sound of other people laughing as 
negative social evaluation, which illustrates how a disorder's specific aspects may be reflected in the cognitive bias content, referred to as content specificity $[30 \bullet, 31]$.

The risk of cognitive biases like HAI is increased by deficits in executive functions like working memory [18•, 32]. When people with impaired working memory process a vignette in which a boy steps on the protagonist's dropped homework while walking past [33], they may struggle to integrate subtle ambiguous intent cues, like the boy looking in a different direction when stepping on the homework, with more salient negative outcome cues like the dirty footprint on the homework $[16 \bullet, 34,35]$. Given that executive functions are frequently impaired in individuals with NDD, particularly in ID, ADHD, and ASD [7, 9, 36], we might expect to find increased HAI in this group. Evidence for the role of HAI in mediating the relation between working memory and aggression [16•] and the relation between victimisation and aggression $[37,38]$ suggests that this cognitive bias may represent an important mechanism linking different risk factors to aggression.

While different types of NDD are difficult to distinguish at the neuropsychological level, more severe problems with Theory of Mind in individuals with ASD [39•] might make this group particularly vulnerable to HAI by making it difficult for them to envision that another person's intent may be non-hostile when the outcome is negative [20, 40]. This might explain the link between Theory of Mind and social anxiety or aggression [41-43].

The role of cognitive biases in explaining the increased risk for mental health problems in ASD was explored by two previous reviews which, however, only identified attention or memory bias studies and provided no evidence to suggest that cognitive biases in CYP or adults with ASD are higher than in TD controls or linked to internalising problems

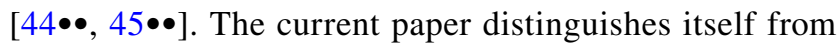
these previous reviews by investigating the role of cognitive biases across all NDD and by integrating findings from the wider cognitive bias field and the SIP field that differ in how they assess and define cognitive bias.

\section{Valence-Specific Processing and Ambiguity of Interpersonal Situations}

While interpretation bias is generally defined as valencespecific (e.g., negative) interpretation of ambiguity [46, 47•], some SIP studies have defined HAI as the attribution of hostility to other people, both when other people's intent is ambiguous and when it varies between being ambiguous, hostile, and non-hostile across presented situations $[25,29 \bullet]$. If a scenario depicts other people's behaviours as non-hostile, for instance indicated by their surprised facial expression or sincere apology, then its hostile interpretation arguably represents an inaccuracy rather than a bias in information processing [48, 49]. In order to distinguish biased from inaccurate processing, the current paper therefore defines cognitive bias as valence-specific processing of interpersonal information that is ambiguous.

A specific focus on the processing of ambiguous information may be important for distinguishing individuals with and without aggression [50]. For instance, adolescents with mild ID were more likely than those with average IQ to feel angry, to evaluate aggressive responses positively and to select aggressive responses, when processing ambiguous scenarios, not when processing accidental or hostile scenarios [51].

\section{Objective}

The primary objective of the current review is to identify whether CYP with NDD show valence-specific processing of interpersonal ambiguous information. As a secondary objective and within the identified evidence base, we aimed to determine whether potential cognitive biases in CYP with NDD can be associated with internalising or externalising problems.

\section{Methods}

\section{Search Strategy}

We conducted a systematic database search in PsycINFO, PsycARTICLES, MEDLINE, Cochrane Central Register of Controlled Trials (CENTRAL), and Science Citation Index using a combination of MeSH terms and synonyms of "children and young people", "neurodevelopmental disorder", "cognitive bias", and "modification". The complete search string can be found in Appendix A.1. Initial searches were conducted on 17.04.2017 and the updated final search was on 05.07.2020. The review protocol was registered on PROSPERO (reference number: CRD42017058346).

\section{Eligibility Criteria}

Inclusion criteria applied to participants, assessment of cognitive biases, and publication characteristics.

\section{Participants}

We included only studies with human participants under the age of 18 with NDD. The list of eligible NDD was drawn up from the index of the Diagnostic and Statistical Manual of Mental Disorders (5th ed.; DSM-5; [5]). For 
each condition identified in this way, studies were allowed to use diagnostic criteria from earlier versions to account for changes in diagnostic criteria over time.

\section{Cognitive Bias}

Three criteria applied to select cognitive biases for this review. Biases eligible for review had to (i) be valencespecific; (ii) concern either interpretation, memory, or attention bias; and (iii) be directed towards ambiguous interpersonal stimuli.

Stimuli were defined as interpersonal when they related to the behaviours or emotions of other people, such as their intent or facial expressions.

Ambiguity referred to the unresolved overall meaning of the stimuli due to the presence of unclear social cues relating to intent or emotion. For example, a scenario in which the protagonist wants to join a game would be unambiguous if his peers gave a neutral reason for not letting him join, such as being told by the teacher that the number of players is fixed [52], but would be ambiguous if peers simply gave no reply, as this would represent an intent that is neither clearly hostile, nor clearly non-hostile. The absence of clear intent then makes the scenario open to being processed in a valence-specific manner, such as interpreting ambiguous information as either positive or negative. In order to qualify as ambiguous, unclear social cues had to exist within the same stimulus (e.g., scenario, image, face, or text). Thus, morphed faces with both negative and positive facial features would meet the ambiguity criterion [53•], while presenting neutral faces next to a negative one would not [54].

For valence specificity, the unclear intent would make a stimulus open to being processed in a valence-specific manner. This refers to the tendency to selectively process the valence of a stimulus. Take the example of an ambiguous scenario with a negative outcome (like a football next to a broken window) which is presented with both hostile and non-hostile social cues relating to intent [22]. In this case, a valence-specific cognitive bias would be a tendency to selectively encode hostile cues (like the person's leg pointing in the direction of the window), while neglecting to encode non-hostile cues (like the person looking into a different direction) [22].

For a study to be eligible for review, its outcome data regarding the processing of ambiguous interpersonal stimuli had to be reported separately and not as an overall bias score including both ambiguous and unambiguous stimuli. This is in contrast to Verhoef et al. who also included studies that used bias measures containing a mixture of ambiguous and unambiguous social situations and that therefore included effect sizes based on composite scores [29•].

\section{Publication Characteristics}

The review included articles in English, Dutch, French, and German from 1980 onwards, as we anticipated a dearth of literature. No limitations were placed on study design. Comparisons of interest were between the different NDD, as well as between CYP with NDD and their TD peers.

\section{Study Selection}

Double reviewing was performed in two steps with an initial cut-off after $30 \%$ of identified records to calculate kappa before proceeding with reviewing the remaining $70 \%$ of articles. Disagreements were resolved through discussion. The same procedures were applied to reviewing the selected full-length articles, with reasons for excluding full-length articles being recorded by each reviewer.

The systematic search was complemented through contact with authors of studies meeting the review eligibility criteria and through use of the ancestry method, whereby references and citations of included studies were checked for potential new studies which the search strategy may have missed.

\section{Outcomes}

The primary outcome of interest was the potential presence and extent of biased processing of interpersonal ambiguous stimuli. To that extent, we extracted data regarding the type of bias (memory, attention, or a specific interpretation bias), assessment method (e.g. questionnaire, vignette, interview), and quantitative outcome data for the relevant bias measure. Included data and summary findings refer only to ambiguous interpersonal stimuli, for which the separate reporting of these outcomes was a requirement to be included in this review. Hence, if studies only presented integrated findings of the processing across ambiguous and unambiguous stimuli, or both interpersonal and non-interpersonal stimuli, then that study would be excluded as it would not be possible to deduct to what extent participants demonstrated biased memory, attention or interpretation specifically for ambiguous interpersonal stimuli. Depending on how the data in the original studies were presented, this could result in data being reported for a single ambiguous interpersonal vignette, for a subset of ambiguous interpersonal stimuli or for a subscale including the ambiguous stimuli.

For secondary outcomes, data was extracted regarding the association of cognitive biases with measures of internalising and externalising problems.

Findings were summarised using a narrative synthesis. 


\section{Quality Assessment}

This study adheres to PRISMA reporting guidelines for systematic reviews [55] (Appendix A.2). Methodological quality and risk of bias of individual studies was evaluated using an adaptation of the Appraisal Tool for Cross-sectional Studies (AXIS; [56]) with additional items from the Cochrane Collaboration guidelines for intervention studies [57]. Potential publication and reporting biases were addressed to assess the risk of bias across studies.

\section{Results}

The initial systematic search yielded 2369 records, whereas the repeated searches on 05.07.2020 to catch studies published since 2016 identified 4220 records. Following removal of duplicates and exclusion of reviews, commentaries, and studies in non-eligible languages, 5499 titles and abstracts were screened for inclusion by both reviewers.

For the initial search, 77 studies proceeded to full-text review by both reviewers. For the first twenty records, all inter-rater disagreements were concerning how primary studies defined or presented ambiguous stimuli. To handle this great variability and lack of clarity in the original studies, we refined our description of the review eligibility criteria. Where the original review protocol already focused on ambiguous stimuli, the text was revised to more clearly define ambiguity and to account for studies that presented both ambiguous and non-ambiguous data. This did not constitute a diversion from the original protocol but rather a further clarification. This clarification was followed by an overall agreement of $K=0.936$ for the remaining 57 studies.

For the most recent searches in 2020 to catch studies published since 2016, an inter-rater agreement of $99.76 \%$ or $K=0.895$ for all articles was observed. Upon removing studies that were already included in the initial search (so published in 2016 or 2017), the reviewers reached an agreement of $99.78 \%$ with 44 agreements and one disagreement. For this step, kappa could not be calculated as reviewer 1 included no studies, which resulted in a lack of variability in the ratings of reviewer 1.

The primary reason for exclusion was that ambiguous stimuli were either absent or not separately reported. Figure 1 shows the combined results of the initial and final database searches and selection processes.

Sixteen studies met all inclusion criteria, of which six addressed interpretation biases in CYP with ID, six in CYP with ADHD, three in ASD, and one in LD. The majority of the identified studies, nearly $70 \%$, addressed hostile attribution of intent (HAI). Table 1 presents study characteristics and outcomes. Despite having a wider search remit to catch attention, memory, and interpretation bias, the search yielded only interpretation bias studies. This was because none of the identified attention or memory bias studies used ambiguous stimuli and therefore had to be excluded. Other studies were excluded for reporting cognitive bias as the combined bias score for ambiguous and unambiguous stimuli. Examples of excluded papers are shown in Appendix A.3.

\section{Study Characteristics}

All sixteen studies assessed interpretation bias using vignettes, but they differed in terms of task design, total number of vignettes, and proportion of ambiguous scenarios. Ten studies measured bias by coding participants' replies to SIP interviews. This involved asking participants open questions to recall and describe what happened in the vignettes or whether they regarded the actions of other people in a particular scenario as mean
Fig. 1 Flow diagram for study selection, following the PRISMA statement
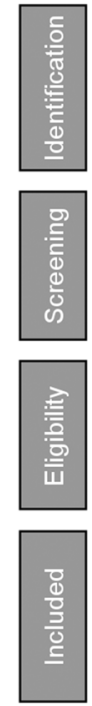

Records identified through database searching, before removing duplicates and commentaries $(n=6589)$

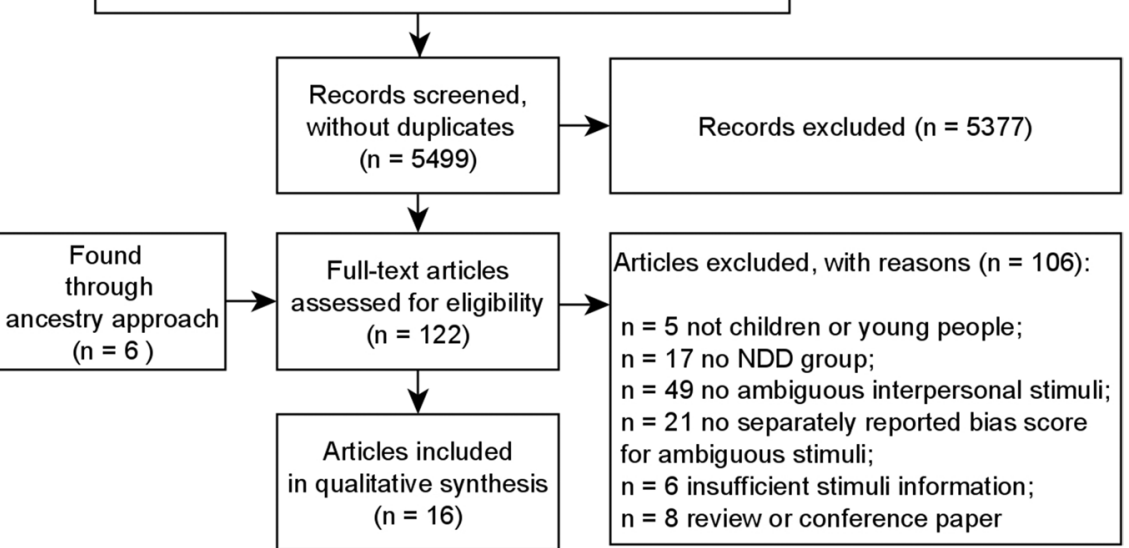




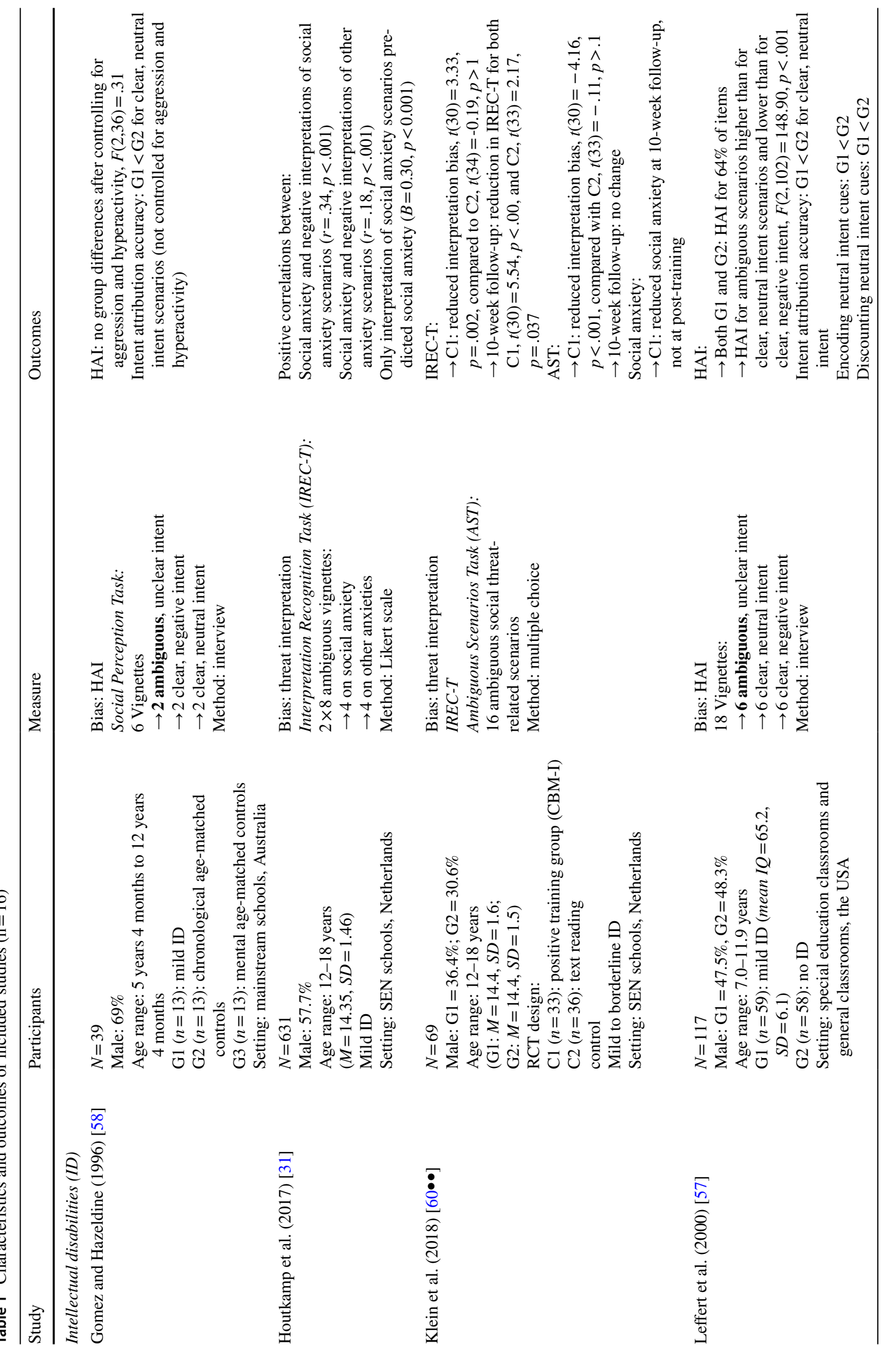




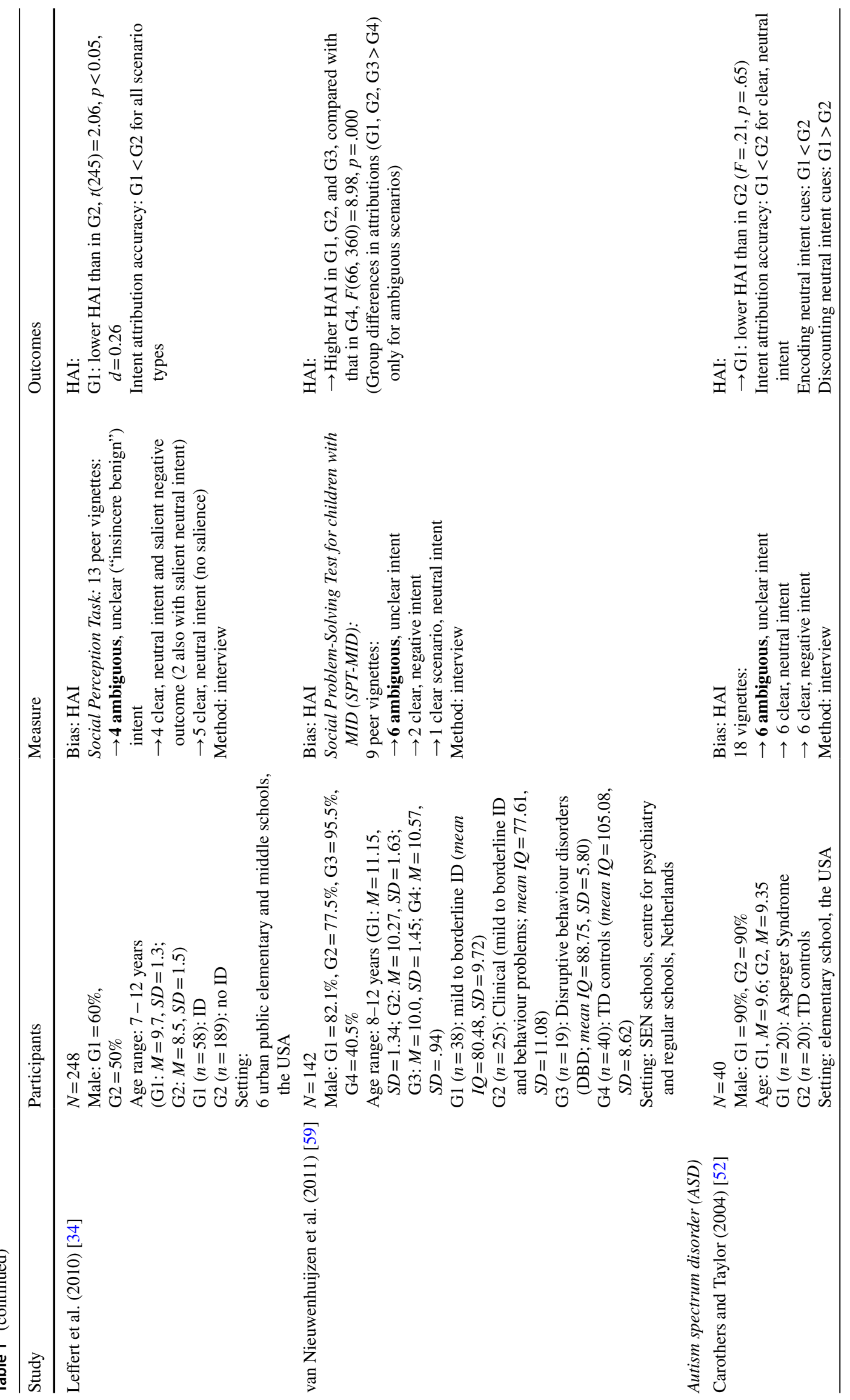




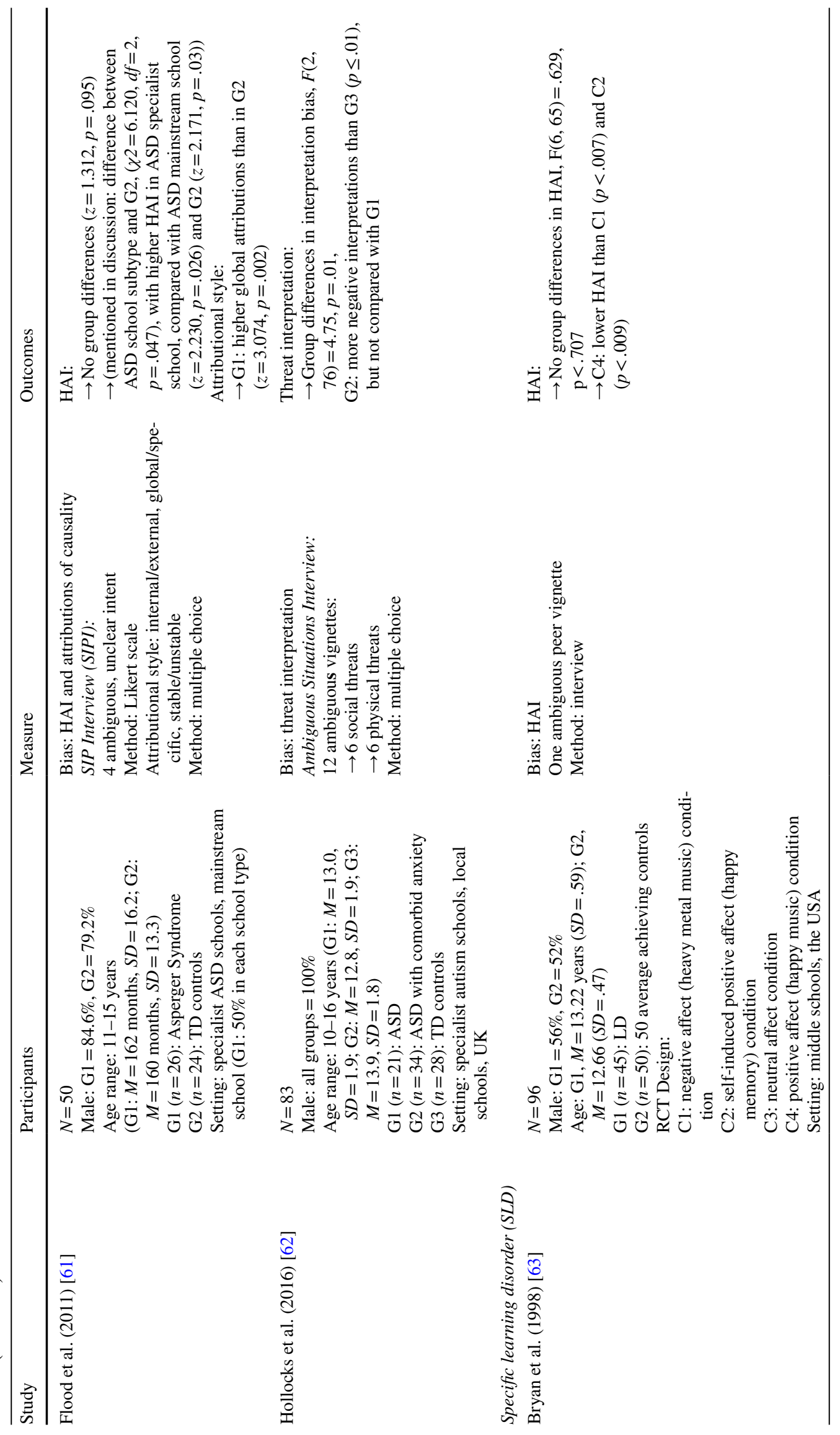




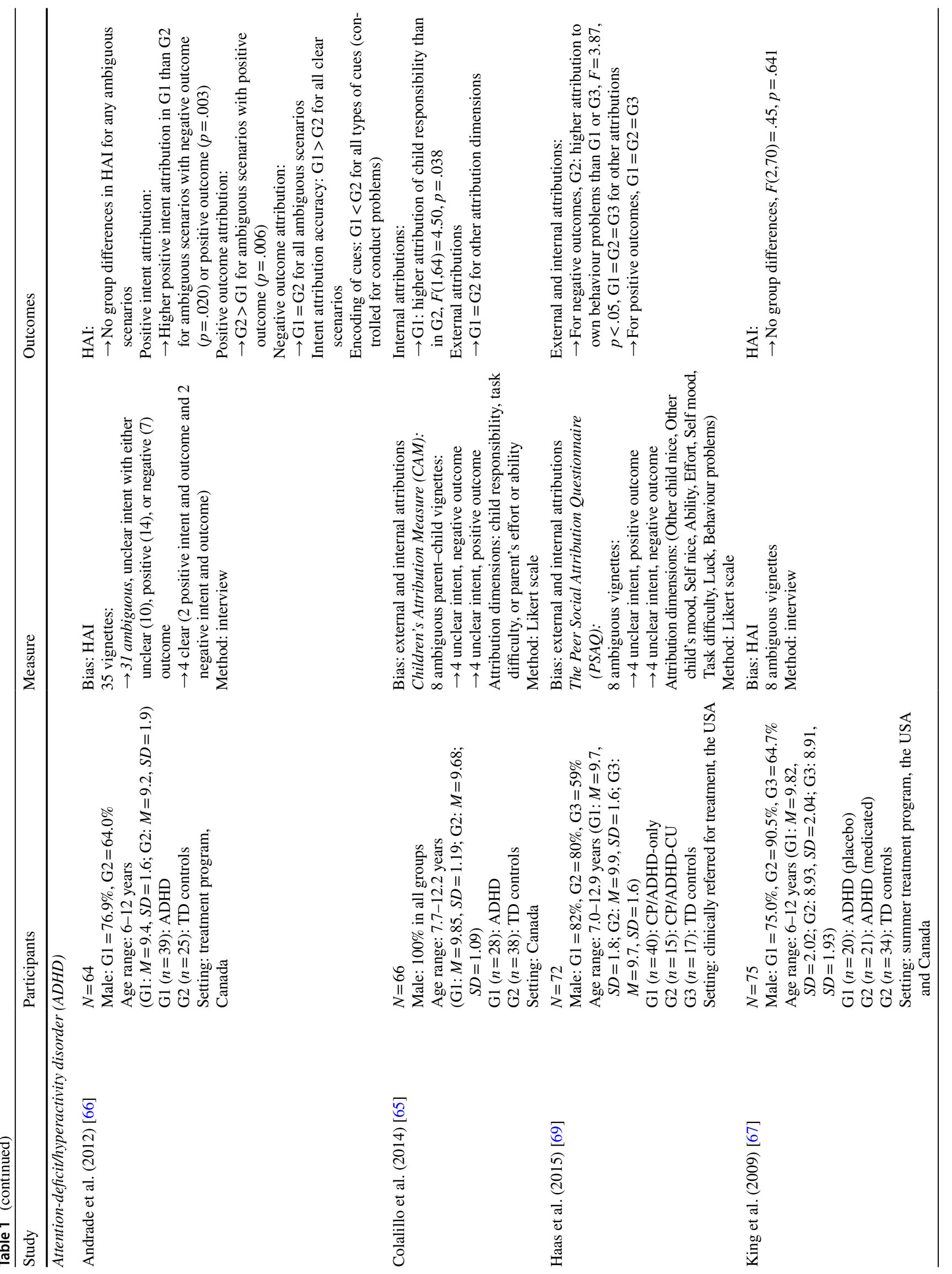




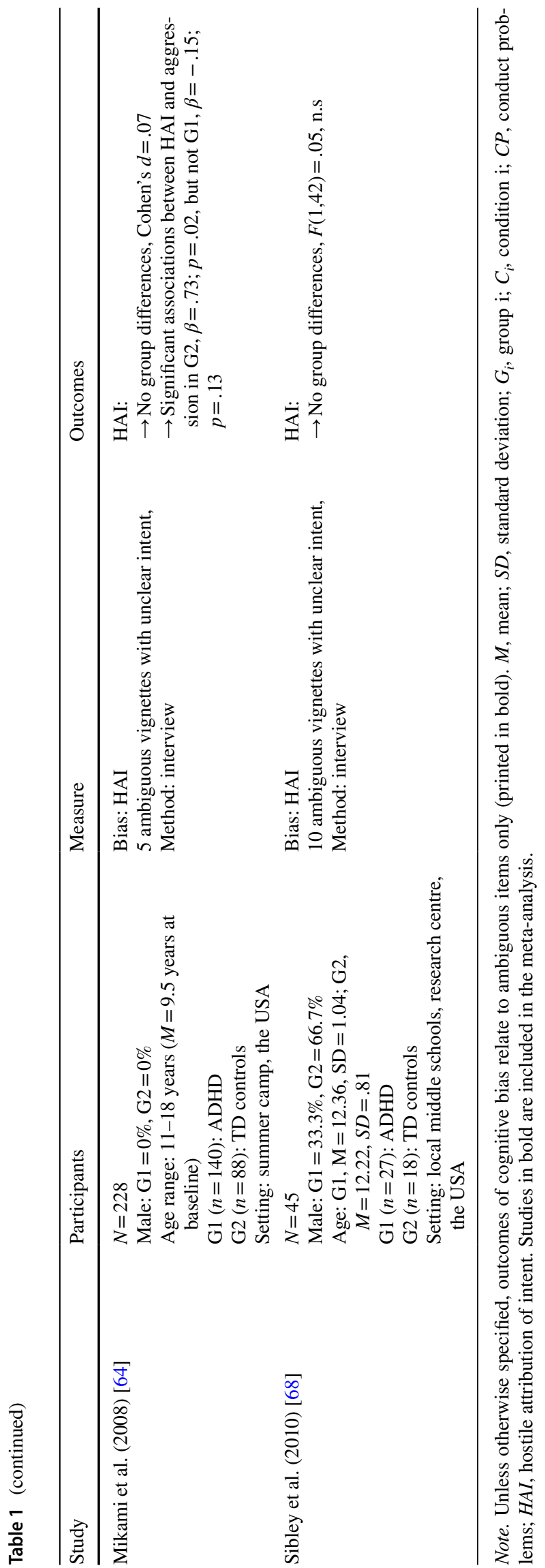

or threatening. Six studies instead assessed bias using forced-choice responding by asking participants to rate their agreement of possible interpretations on a Likert scale or to choose one of multiple interpretations. Across studies, the number of ambiguous vignettes ranged from one to 31 , with a mean of $7.31(S D=6.750)$, and most studies either adapted previous bias measures or developed new ones.

While there were no substantial differences in risk of bias between the studies, Table 2 demonstrates that potential sources of bias are due to the absence of a sample size justification and insufficient information about response rates and nonresponders.

The narrative synthesis of these studies is structured according to population characteristics and strength of the evidence, thereby integrating the findings of the quality assessment (Table 2).

\section{Interpretation Bias and Intellectual Disabilities}

Six studies assessed interpretation bias in ID. One study reported that HAI in children with and without mild ID was higher for ambiguous than for accidental vignettes, although no exact statistics were provided [57]. Compared with controls, children with ID showed less hostile interpretations of potential insincerity, such as someone's ambiguously sincere apology [34]. The only gender-balanced but smallest of the six studies, restricted participation to those with sufficient verbal skills and found no HAI differences between CYP with mild ID and matched controls [58]. In a sample that was 71.6\% male, higher HAI was found for children with ID, behaviour problems, or both, when compared with TD controls [59].

In contrast to these four studies assessing HAI using open questions, two high-quality studies assessed threat interpretations using a Likert scale measure, namely the Interpretation Recognition Task (IREC-T). Houtkamp et al. found that social anxiety in 631 young people with mild ID was significantly predicted by negative interpretations of scenarios relating to social anxiety, as opposed to other anxieties [31]. Using a subsample of this study, threat interpretation biases were reduced following a 3-week positive cognitive bias modification training for interpretations (CBM-I), relative to a text-reading control involving non-emotional unambiguous scenarios [60••]. While IREC-T scores were also decreased at 10-week follow-up in the control group, only the positive CBM-I group showed reduced social anxiety symptoms at follow-up. 


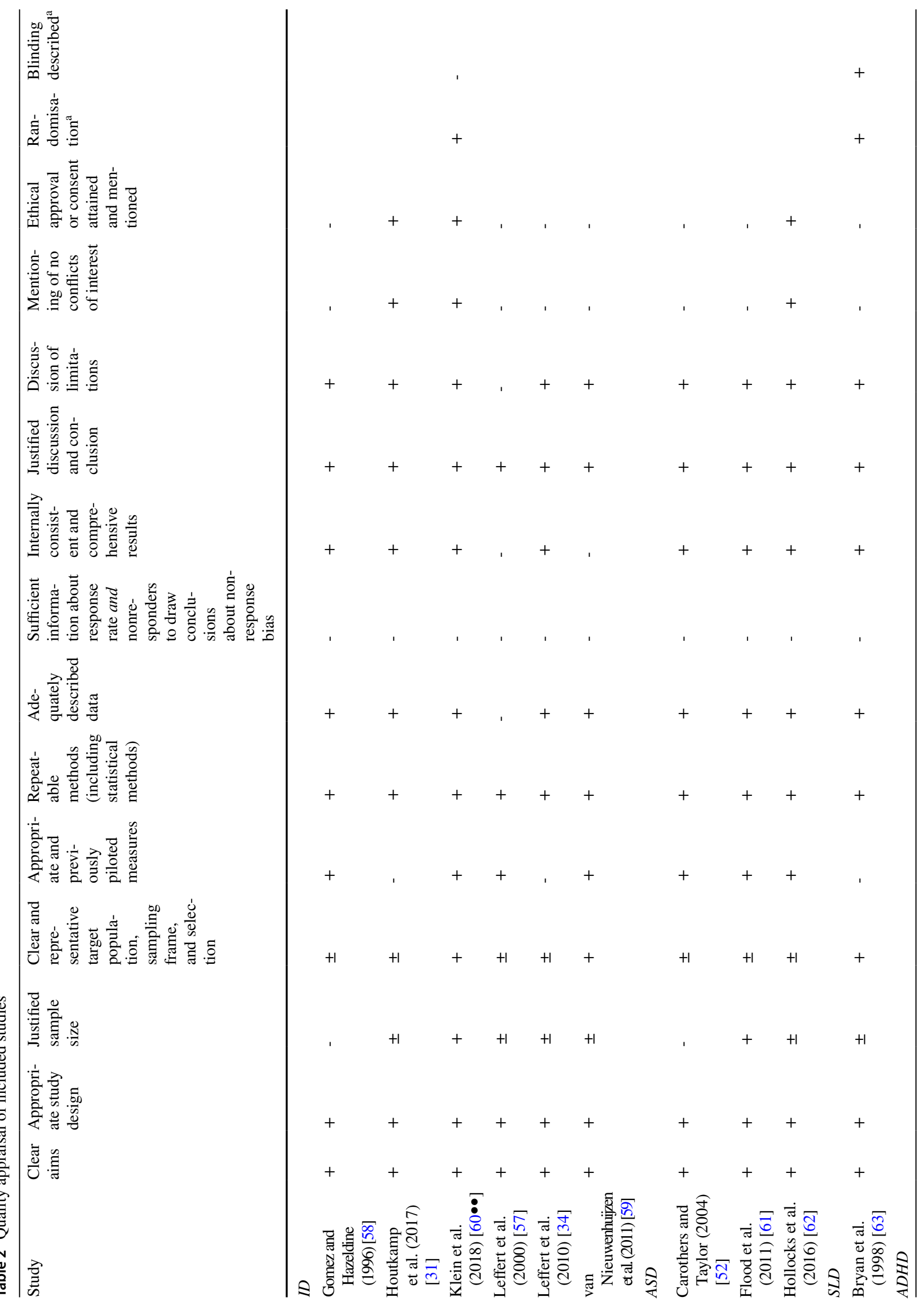




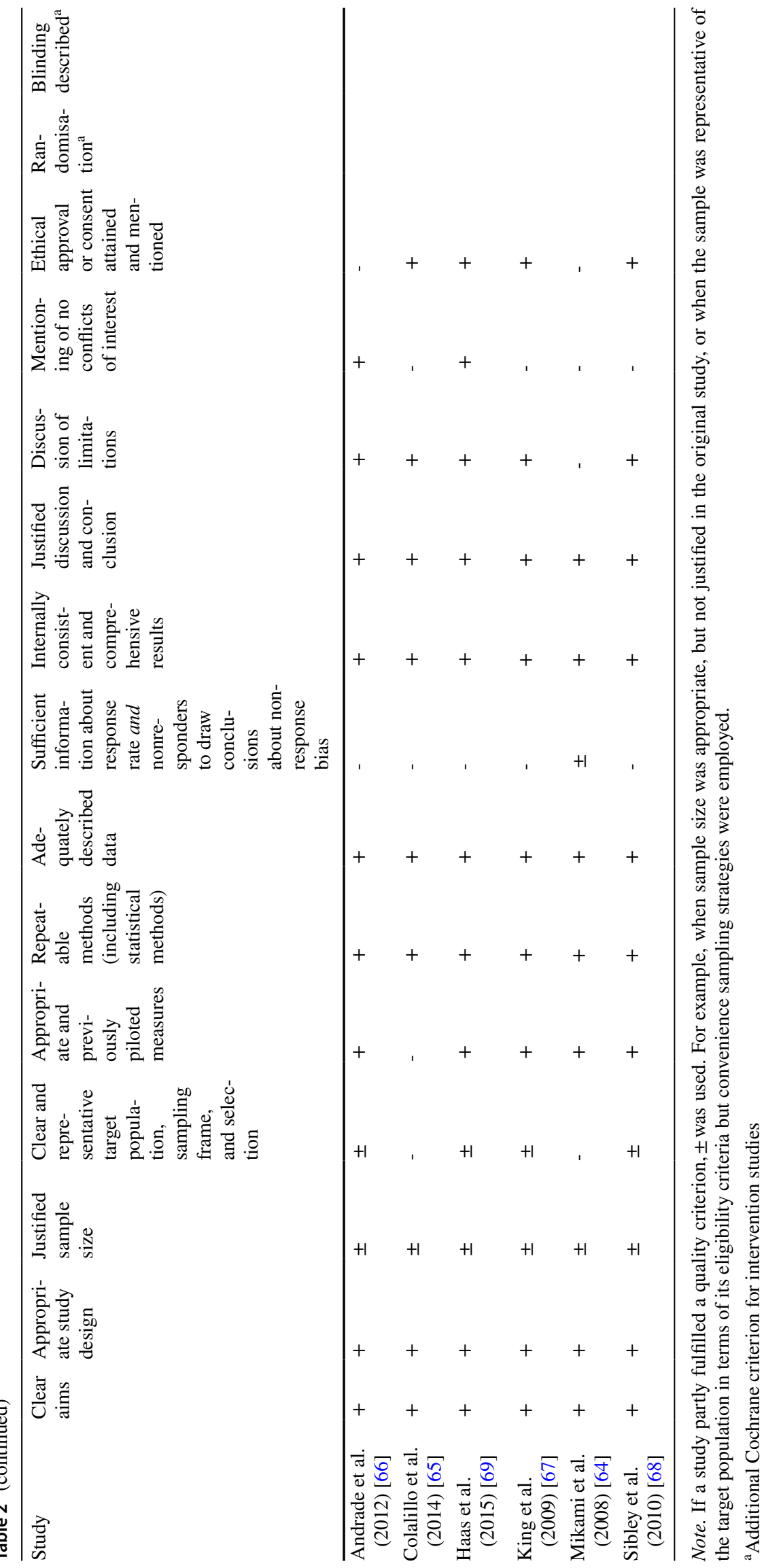




\section{Interpretation Bias and Autism Spectrum Disorder}

Three studies assessed cognitive bias in CYP with ASD and had predominantly male samples. Carothers and Taylor compared twenty children with Asperger syndrome (AS) with twenty TD peers and reported lower HAI in the former [52]. Excluding participants with dual diagnoses of other NDD like ADHD or learning disability, Flood et al. found that children with AS did not differ from controls in HAI or internality and stability of attributions, but made more global than specific attributions [61]. Boys with ASD, compared with those without ASD, held more negative interpretations of social threat situations, as demonstrated in the methodologically strongest study, however using an all-male sample [62].

\section{Interpretation Bias and Specific Learning Disorder}

Bryan et al. present the only study including CYP with SLD which was eligible for this review and reported no differences in HAI between participants with and without SLD [63]. Inducing positive affect through happy music did lower HAI, relative to inducing a negative affect through music and relative to positive memory recall.

\section{Interpretation Bias and Attention-Deficit/ Hyperactivity Disorder}

Six studies assessed interpretation bias in CYP with ADHD, including one study with an all-male sample, and one with an all-female sample. Adolescent girls with ADHD, recruited through a research summer camp, did not differ in HAI from female controls and, unlike controls, showed no association of HAI with aggression [64]. Likewise, boys with ADHD did not differ from controls in their external attributions of parental behaviour, but made more internal attributions to child responsibility [65]. Three gender-mixed studies that directly compared HAI between CYP with and without ADHD failed to find group differences for HAI [66-68]. Finally, children with comorbid ADHD, conduct problems and callous-unemotional traits did not differ from those without callous-unemotional traits or from TD controls in external attributions, but instead were more likely to attribute negative outcomes to own behaviour problems [69].

\section{Additional Exploration}

Considering the studies' data relating to the accuracy of processing neutral stimuli, in contrast to ambiguous stimuli, we found that CYP with ID [34, 58] or AS [52] were less accurate at interpreting neutral intent and encoded less neutral cues than controls. The latter was also found in children with ADHD, who encoded fewer cues, irrespective of cue valence [66]. When rejecting neutral cues, children with ID did this at a lower rate [57], and children with AS at a higher rate [52] than controls.

\section{Discussion}

Our primary objective was to examine the evidence regarding biased processing of ambiguous interpersonal information in CYP with NDD. In contrast to the extensive cognitive bias research literature relating to TD individuals, only 16 studies met our eligibility criteria, all of which focused on interpretation bias and not on memory or attention bias. The majority of identified studies assessed whether participants with NDD were more likely than controls to interpret others' behaviours as more hostile. Only one study found evidence for higher HAI in children with mild to borderline ID [59], in line with a study that emerged since the systematic review's completion which reported higher HAI in the low IQ group, relative to the average IQ group [51]. However, contrary to our hypotheses, overall evidence for increased HAI in NDD was inconsistent. By contrast, there were indications that attributions of causality differed between CYP with and without NDD, as shown by increased globality of attributions in AS [61], or internality of attributions in CYP with ADHD [65].

In light of our second objective regarding mental health outcomes, HAI was expected to be associated with aggression in NDD. However, the only identified study that assessed such associations found HAI to be associated with aggression only in girls without ADHD, not in girls with ADHD [64]. Meanwhile, evidence for biases associated with internalising disorders in individuals with NDD was more consistent. Houtkamp et al. found a content-specific interpretation bias associated with social anxiety in CYP with mild ID, whose biases and symptoms were improved through positive CBM-I [31, 60••]. Hollocks et al. (2016) finding that interpretations of ambiguous social threat scenarios were more negative in CYP with ASD and comorbid anxiety than in TD controls indicates that biased ambiguity processing might also be a suitable CBM-I target for anxiety in individuals with ASD [62].

Although we focused on the distorted processing of ambiguous information, some of the included studies presented separate analyses for ambiguity processing (as per our inclusion criterion), but generally defined cognitive bias more broadly to also include unambiguous stimuli. An additional exploration of these studies' findings showed that, compared with controls, CYP with ID and ASD were less accurate at interpreting scenarios with clearly accidental intent and at encoding neutral cues [34, 52, 58]. While we acknowledge that such deficits in encoding and interpreting unambiguous information are also important for 
understanding the cognitive processes associated with specific NDD, the ambiguity criterion was essential to our study because it enables us to distinguish biased from erroneous processing [48-50].

The ambiguity criterion was also essential in our study because of its potential to help understand psychiatric comorbidities. Initial evidence for this was found in both CYP with mild ID [31] and ASD [62]. The proposed link between biased ambiguity processing and mental health in NDD was further supported by evidence that specifically targeting interpretations of ambiguous scenarios through CBM-I improved anxiety in people with mild ID [60••].

As a result of our ambiguity criterion for cognitive bias, we excluded a large body of evidence that only used unambiguous stimuli, such as studies assessing attention bias in CYP with NDD. While it might therefore be regarded as a limitation of this review that we did not cover all possible available cognitive bias research in NDD, it is important to note that our search strategy was inclusive (see Appendix A.1). Our ambiguity criterion did not per se exclude attention bias studies, because it is possible to study attention bias with ambiguous stimuli, such as through tracking eye movements to social cues in ambiguous scenarios [22, 70] or in ambiguous facial expressions [71]. Nevertheless, without our ambiguity criterion, we could have reviewed more research and could have drawn conclusions about the role of attention bias in NDD.

Since evidence for the role of cognitive bias in NDD was inconsistent in the current review and in previous reviews

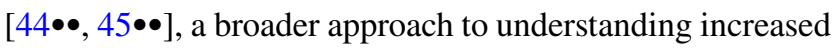
aggression in NDD might be to consider deficits in later stages of the SIP model. For instance, the tendency to generate and feel confident about aggressive responses moderated the relation between working memory and aggression in CYP with mild ID [16•] and was found to be higher in individuals with ID compared with controls [51, 57-59]. While increased rates of aggression in NDD might therefore partly be due to an increased generation and positive evaluation of aggressive responses, it might also be linked to impairments in the generation of competent responses, which were identified in CYP with ID [57, 59, 72] and in CYP with ASD [73-75]. Since the effect of an aggression prevention training on antisocial behaviour was mediated by its impact on children's ability to generate competent responses, as well as by its impact on HAI and aggressive response evaluation, all three steps may represent causes of aggression and therefore important treatment targets [76].

\section{Clinical Implications}

For clinicians, the findings of this review indicate that interpretation biases represent a potential mechanism involved in the occurrence of aggression or internalising problems in individuals with NDD.

Most evidence of this review relates to hostile intent attributions in CYP with ADHD or ID, and it was found that such attributions were not consistently higher in the NDD groups than in controls. Meanwhile, little is known about the association of HAI and aggression in CYP with ADHD. Consequently, one might consider ADHD and aggression as distinct dimensions of externalising problems [10, 77], each with their different intervention and support options. Furthermore, given that aggression is robustly linked to HAI in TD populations [29•] and that it can be reduced using interventions that target HAI [78•], assessing and modifying HAI in CYP with ADHD could help researchers and clinicians to understand and reduce these individuals' psychosocial problems that are likely exacerbated by their frequent comorbidity with aggression.

Finally, if future research replicates our tentative findings regarding increased internal or global attributions in CYP with ADHD or ASD, and for links between social threat interpretation bias and anxiety in ASD and mild ID, this would support the assessment and modification of such biases to alleviate mental health problems in this group.

\section{Conclusions}

Only a small number of studies investigated interpersonal cognitive biases in CYP with NDD, despite the potential importance of such biases in understanding the increased risk of developing mental health problems in this population. There was some evidence to suggest that threat interpretation bias may explain anxiety in CYP with ID or ASD and represent a suitable intervention target for CBM-I in these groups. Regardless of whether future studies find cognitive bias to differ between CYP with NDD and TD individuals, the inclusion of both groups in CBM is necessary to make samples more representative and the frequent categorical exclusion of this group from cognitive bias research needs to be justified [79•].

Supplementary Information The online version contains supplementary material available at https://doi.org/10.1007/s40474-021-00239-9.

Acknowledgements We thank Alina Simonsen for assistance with data extraction and quality assessment.

Funding Open Access funding enabled and organized by Projekt DEAL.

\section{Declarations}

Financial Interest or Conflict of Interest Nothing to declare. 
Human and Animal Rights This article does not contain any studies with human or animal subjects performed by any of the authors.

Open Access This article is licensed under a Creative Commons Attribution 4.0 International License, which permits use, sharing, adaptation, distribution and reproduction in any medium or format, as long as you give appropriate credit to the original author(s) and the source, provide a link to the Creative Commons licence, and indicate if changes were made. The images or other third party material in this article are included in the article's Creative Commons licence, unless indicated otherwise in a credit line to the material. If material is not included in the article's Creative Commons licence and your intended use is not permitted by statutory regulation or exceeds the permitted use, you will need to obtain permission directly from the copyright holder. To view a copy of this licence, visit http://creativecommons.org/licenses/by/4.0/.

\section{References}

Papers of particular interest, published recently, have been highlighted as:

- Of importance

$\bullet$ Of major importance

1. Emerson E. Prevalence of psychiatric disorders in children and adolescents with and without intellectual disability. J Intellect Disabil Res. 2003;47:51-8. https://doi.org/10.1046/j.13652788.2003.00464.x.

2. Hansen BH, Oerbeck B, Skirbekk B, Petrovski BÉ, Kristensen $\mathrm{H}$. Neurodevelopmental disorders: prevalence and comorbidity in children referred to mental health services. Nord J Psychiatry. 2018;72:285-91. https://doi.org/10.1080/08039488.2018. 1444087.

3. Simonoff E, Pickles A, Charman T, Chandler S, Loucas T, Baird G. Psychiatric disorders in children with autism spectrum disorders: prevalence, comorbidity, and associated factors in a population-derived sample. J Am Acad Child Adolesc Psychiatry. 2008;47:921-9. https://doi.org/10.1097/CHI.0b013 e318179964f.

4. Taurines R, Schmitt J, Renner T, Conner AC, Warnke A, Romanos M. Developmental comorbidity in attention-deficit/hyperactivity disorder. Atten Defic Hyperact Disord. 2010;2:267-89. https://doi.org/10.1007/ s12402-010-0040-0.

5. American Psychiatric Association. Diagnostic and statistical manual of mental disorders (5th ed.). Washington, DC. 2013. https://doi.org/10.1176/appi.books. 9780890425596

6. Cleaton MAM, Kirby A. Why do we find it so hard to calculate the burden of neurodevelopmental disorders? J Child Dev Disord. 2018. https://doi.org/10.4172/2472-1786.100073.

7. Craig F, Margari F, Legrottaglie AR, Palumbi R, de Giambattista C, Margari L. A review of executive function deficits in autism spectrum disorder and attention-deficit/hyperactivity disorder. Neuropsychiatr Dis Treat. 2016;12:1191-202. https:// doi.org/10.2147/NDT.S104620.

8. England-Mason G. Emotion regulation as a transdiagnostic feature in children with neurodevelopmental disorders. Curr Dev Disord Rep. 2020;7:130-8. https://doi.org/10.1007/ s40474-020-00200-2.

9. van der Molen MJ, van Luit JEH, Jongmans MJ, van der Molen MW. Verbal working memory in children with mild intellectual disabilities. J Intellect Disabil Res. 2007;51:162-9. https://doi.org/10.1111/j.1365-2788.2006.00863.x.
10. King S, Waschbusch DA. Aggression in children with attention-deficit/hyperactivity disorder. Expert Rev Neurother. 2010;10:1581-94. https://doi.org/10.1586/ern.10.146.

11. Fitzpatrick SE, Srivorakiat L, Wink LK, Pedapati EV, Erickson CA. Aggression in autism spectrum disorder: presentation and treatment options. Neuropsychiatr Dis Treat. 2016;12:152538. https://doi.org/10.2147/NDT.S84585.

12. Dekker MC, Koot HM, van der Ende J, Verhulst FC. Emotional and behavioral problems in children and adolescents with and without intellectual disability. J Child Psychol \& Psychiat. 2002;43:1087-98. https://doi.org/10.1111/1469-7610.00235.

13. Rodriguez MC, Wade TJ, Veldhuizen S, Missiuna C, Timmons B, Cairney J. Emotional and behavioral problems in 4- and 5 -year old children with and without motor delays. Front Pediatr. 2019;7:474. https://doi.org/10.3389/fped.2019.00474.

14. van Nieuwenhuijzen M, Vriens A. (Social) Cognitive skills and social information processing in children with mild to borderline intellectual disabilities. Res Dev Disabil. 2012;33:426-34. https://doi.org/10.1016/j.ridd.2011.09.025.

15. van Nieuwenhuijzen M, Orobio de Castro B, van der Valk I, Wijnroks L, Vermeer A, Matthys W. Do social informationprocessing models explain aggressive behaviour by children with mild intellectual disabilities in residential care? J Intellect Disabil Res. 2006;50:801-12. https://doi.org/10.1111/j.13652788.2005.00773.x.

16. van Rest MM, Matthys W, van Nieuwenhuijzen M, de Moor $\mathrm{MH}$, Vriens A, Schuengel C. Social information processing skills link executive functions to aggression in adolescents with mild to borderline intellectual disability. Child Neuropsychol. 2019;25:573-98. https://doi.org/10.1080/09297 049.2018.1495186 (Assessing the role of SIP in explaining the relation between executive functions and aggression in CYP with mild to borderline ID, this paper showed that HAI was one of the SIP steps that mediated the link between both focused attention and working memory, and aggression. This indicates that cognitive bias can help explain aggression in this NDD group).

17. Crick NR, Dodge KA. A review and reformulation of social information-processing mechanisms in children's social adjustment. Psychol Bull. 1994;115:74-101. https://doi.org/ 10.1037//0033-2909.115.1.74.

18. Garrigan B, Adlam AL, Langdon PE. Moral decision-making and moral development: toward an integrative framework. Dev Rev. 2018;49:80-100. https://doi.org/10.1016/j.dr.2018.06. 001 (These authors developed a framework that integrates the social information processing model with theories about moral development and that takes into account the influence of developmental factors like executive functions and ToM, which helps explain decision-making in individuals with or without NDD).

19. Larkin P, Jahoda A, MacMahon K. The social information processing model as a framework for explaining frequent aggression in adults with mild to moderate intellectual disabilities: a systematic review of the evidence. J Appl Res Intellect Disabil. 2013;26:447-65. https://doi.org/10.1111/jar.12031.

20. Chester V, Langdon PE. The clinical utility of social information processing theory in assessing and treating offenders with autism spectrum disorder. Adv Autism. 2016;2:154-71. https://doi.org/10.1108/AIA-07-2016-0019.

21 Horsley TA, Orobio de Castro B, van der Schoot M. In the eye of the beholder: eye-tracking assessment of social information processing in aggressive behavior. $\mathrm{J}$ Abnorm Child Psychol. 2010;38:587-99. https://doi.org/10.1007/ s10802-009-9361-x.

22. Wilkowski BM, Robinson MD, Gordon RD, Troop-Gordon W. Tracking the evil eye: trait anger and selective attention 
within ambiguously hostile scenes. J Res Pers. 2007;41:65066. https://doi.org/10.1016/j.jrp.2006.07.003.

23. Platt B, Waters AM, Schulte-Koerne G, Engelmann L, Salemink E. A review of cognitive biases in youth depression: attention, interpretation and memory. Cogn Emot. 2017;31:462-83. https://doi.org/10.1080/02699931.2015. 1127215.

24. Troop-Gordon W, Gordon RD, Vogel-Ciernia L, Ewing Lee E, Visconti KJ. Visual attention to dynamic scenes of ambiguous provocation and children's aggressive behavior. J Clin Child Adolesc Psychol. 2018;47:925-40. https://doi.org/10.1080/ 15374416.2016.1138412.

25 Orobio de Castro B, Veerman JW, Koops W, Bosch JD, Monshouwer HJ. Hostile attribution of intent and aggressive behavior: a meta-analysis. Child Dev. 2002;73:916-34. https://doi.org/ 10.1111/1467-8624.00447.

26. Lau JYF, Waters AM. Annual research review: an expanded account of information-processing mechanisms in risk for child and adolescent anxiety and depression. J Child Psychol Psychiatry. 2017;58:387-407. https://doi.org/10.1111/jcpp.12653.

27. Mobini S, Reynolds S, Mackintosh B. Clinical implications of cognitive bias modification for iInterpretative biases in social anxiety: an integrative literature review. Cogn Ther Res. 2013;37:173-82. https://doi.org/10.1007/ s10608-012-9445-8.

28 Bar-Haim Y, Lamy D, Pergamin L, Bakermans-Kranenburg MJ, van IJzendoorn MH. Threat-related attentional bias in anxious and nonanxious individuals: a meta-analytic study. Psychol Bull. 2007;133:1-24. https://doi.org/10.1037/0033-2909.133.1.1.

29 Verhoef REJ, Alsem SC, Verhulp EE, Orobio de Castro B. Hostile intent attribution and aggressive behavior in children revisited: a meta-analysis. Child Dev. 2019;90:e525-47. https:// doi.org/10.1111/cdev.13255 (This paper reviewed 111 studies and found that the association between HAI and aggression in children was positive and modest (mean effect size $\mathbf{d}=$ 0.33) and stronger for severely aggressive individuals, but not stronger for reactive aggression or for samples that included CYP with ADHD).

30. Mobach L, Rinck M, Becker ES, Hudson JL, Klein AM. Content-specific interpretation bias in children with varying levels of anxiety: the role of gender and age. Child Psychiatry Hum Dev. 2019;50:803-14. https://doi.org/10.1007/s10578-019-00883-8. (This study illustrates content-specificity of cognitive bias by showing that the content of interpretation bias is specific to children's particular type of fear).

31. Houtkamp EO, van der Molen MJ, de Voogd EL, Salemink E, Klein AM. The relation between social anxiety and biased interpretations in adolescents with mild intellectual disabilities. Res Dev Disabil. 2017;67:94-8. https://doi.org/10.1016/j.ridd.2017. 06.003 .

32. Baddeley A. The episodic buffer: a new component of working memory? Trends Cogn Sci. 2000;4:417-23. https://doi.org/10. 1016/S1364-6613(00)01538-2.

33. Hudley C, Graham S. An attributional intervention to reduce peer-directed aggression among African-American boys. Child Dev. 1993;64:124. https://doi.org/10.2307/1131441.

34. Leffert JS, Siperstein GN, Widaman KF. Social perception in children with intellectual disabilities: the interpretation of benign and hostile intentions. J Intellect Disabil Res. 2010;54:168-80. https://doi.org/10.1111/j.1365-2788.2009.01240.x.

35. Klingberg T. Training and plasticity of working memory. Trends Cogn Sci. 2010;14:317-24. https://doi.org/10.1016/j.tics.2010. 05.002 .

36. Willcutt EG, Doyle AE, Nigg JT, Faraone SV, Pennington BF. Validity of the executive function theory of attention-deficit/ hyperactivity disorder: a meta-analytic review. Biol Psychiatry.
2005;57:1336-46. https://doi.org/10.1016/j.biopsych.2005.02. 006.

37. Perren S, Ettekal I, Ladd G. The impact of peer victimization on later maladjustment: mediating and moderating effects of hostile and self-blaming attributions. J Child Psychol Psychiatry. 2013;54:46-55. https://doi.org/10.1111/j.1469-7610.2012. 02618.x.

38. Dodge KA, Lansford JE, Burks VS, Bates JE, Pettit GS, Fontaine $\mathrm{R}$, Price JM. Peer rejection and social information-processing factors in the development of aggressive behavior problems in children. Child Dev. 2003;74:374-93. https://doi.org/10.1111/ 1467-8624.7402004.

39. Mikami AY, Miller M, Lerner MD. Social functioning in youth with attention-deficit/hyperactivity disorder and autism spectrum disorder: transdiagnostic commonalities and differences. Clin Psychol Rev. 2019;68:54-70. https://doi.org/10.1016/j.cpr. 2018.12.005 (This review shows that children with ADHD display behaviour problems in real world peer situations, not on lab-based tasks, while children with ASD were more likely to be impaired in their social cognition, positive social behaviours and both their knowledge and performance of correct behaviours).

40. Choe DE, Lane JD, Grabell AS, Olson SL. Developmental precursors of young school-age children's hostile attribution bias. Dev Psychol. 2013;49:2245-56. https://doi.org/10.1037/a0032 293.

41. Renouf A, Brendgen M, Séguin JR, Vitaro F, Boivin M, Dionne $\mathrm{G}$, et al. Interactive links between theory of mind, peer victimization, and reactive and proactive aggression. $\mathrm{J}$ Abnorm Child Psychol. 2010;38:1109-23. https://doi.org/10.1007/ s10802-010-9432-z.

42. Colonnesi C, Nikolić M, de Vente W, Bögels SM. Social anxiety symptoms in young children: investigating the interplay of theory of mind and expressions of shyness. $\mathrm{J}$ Abnorm Child Psychol. 2017;45:997-1011. https://doi.org/10.1007/ s10802-016-0206-0.

43. Carter Leno V, Chandler S, White P, Yorke I, Charman T, Jones CRG, et al. Associations between theory of mind and conduct problems in autistic and nonautistic youth. Autism Res. 2021;14:276-88. https://doi.org/10.1002/aur.2346.

44•• Fan X-Z, Duan Y-W, Yi L-X, He H-Z. Attentional bias toward threatening emotional faces in individuals with autism spectrum disorder: a meta-analysis on reaction time tasks. Res Autism Spectr Disord. 2020;78:101646. https://doi.org/10.1016/j.rasd. 2020.101646 (This review identified 21 attention bias studies which did not find increased bias in CYP and adults relative to TD controls, but found that both groups showed an attentional bias towards threatening faces over happy faces for schematic faces and short presentation times. Studies rarely assessed links between attention bias and anxiety).

45.•• Bergman MA, Schene AH, Vissers CT, Vrijsen JN, Kan CC, van Oostrom I. Systematic review of cognitive biases in autism spectrum disorders: a neuropsychological framework towards an understanding of the high prevalence of co-occurring depression. Res Autism Spectr Disord. 2020;69:101455. https://doi. org/10.1016/j.rasd.2019.101455. (The 31 attention bias and memory bias studies identified by this systematic review did not find cognitive bias to be higher in children and adults with ASD than in TD controls or to be linked to internalising problems. However, studies were heterogeneous, did not focus on ambiguity processing and did not address interpretation bias).

46. Schoth DE, Liossi C. A systematic review of experimental paradigms for exploring biased interpretation of ambiguous information with emotional and neutral associations. Front Psychol. 2017;8:171. https://doi.org/10.3389/fpsyg.2017.00171. 
47. Stuijfzand S, Creswell C, Field AP, Pearcey S, Dodd H. Research review: is anxiety associated with negative interpretations of ambiguity in children and adolescents? A systematic review and meta-analysis. J Child Psychol Psychiatry. 2018;59:1127-42. https://doi.org/10.1111/jcpp.12822. (This paper reviewed 77 studies and found a medium effect size for the association between anxiety and negative interpretations of ambiguity in CYP $(\mathrm{d}=\mathbf{0 . 3 3})$. Cognitive bias showed content-specificity and increased with age).

48. Dodge KA. Translational science in action: hostile attributional style and the development of aggressive behavior problems. Dev Psychopathol. 2006;18:791-814. https://doi.org/10.1017/S0954 579406060391.

49. Dodge KA, Murphy RR, Buchsbaum K. The assessment of intention-due detection skills in children: implications for developmental psychopathology. Child Dev. 1984:163-73. https://doi. org/10.2307/1129842.

50. Dodge KA. Social cognition and children's aggressive behavior. Child Dev. 1980;51:162. https://doi.org/10.2307/1129603.

51. van Rest MM, van Nieuwenhuijzen M, Kupersmidt JB, Vriens A, Schuengel C, Matthys W. Accidental and ambiguous situations reveal specific social information processing biases and deficits in adolescents with low intellectual level and clinical levels of externalizing behavior. J Abnorm Child Psychol. 2020;48:141124. https://doi.org/10.1007/s10802-020-00676-X.

52. Carothers DE, Taylor RE. Social cognitive processing in elementary school children with Asperger syndrome. Educ Train Autism Dev Disabil. 2004:177-187. https://www.jstor.org/stable/23880065.

53• Hiemstra W, Orobio de Castro B, Thomaes S. Reducing aggressive children's hostile attributions: a cognitive bias modification procedure. Cogn Ther Res. 2019;43:387-98. https://doi. org/10.1007/s10608-018-9958-x (An intervention study that provided evidence for the effectiveness of CBM-I in reducing hostile interpretation of ambiguous faces, but not aggression, in a sample of aggressive boys who predominantly had ADHD or ASD).

54. García-Blanco A, López-Soler C, Vento M, García-Blanco MC, Gago B, Perea M. Communication deficits and avoidance of angry faces in children with autism spectrum disorder. Res Dev Disabil. 2017;62:218-26. https://doi.org/10.1016/j.ridd.2017.02. 002

55. Moher D, Liberati A, Tetzlaff J, Altman DG. Preferred reporting items for systematic reviews and meta-analyses: the PRISMA statement. PLoS Med. 2009;6: e1000097. https://doi.org/10. 1371/journal.pmed.1000097.

56. Higgins JPT, Altman DG, Gøtzsche PC, Jüni P, Moher D, Oxman AD, et al. The Cochrane Collaboration's tool for assessing risk of bias in randomised trials. BMJ. 2011;343: d5928. https://doi.org/10.1136/bmj.d5928.

57. Leffert JS, Siperstein GN, Millikan E. Understanding social adaptation in children with mental retardation: a social-cognitive perspective. Except Child. 2000;66:530-45. https://doi.org/10. 1177/001440290006600406.

58. Gomez R, Hazeldine P. Social information processing in mild mentally retarded children. Res Dev Disabil. 1996;17:217-27. https://doi.org/10.1016/0891-4222(96)00005-4.

59. van Nieuwenhuijzen M, Vriens A, Scheepmaker M, Smit M, Porton $\mathrm{E}$. The development of a diagnostic instrument to measure social information processing in children with mild to borderline intellectual disabilities. Res Dev Disabil. 2011;32:358-70. https://doi.org/10.1016/j.ridd.2010.10.012.
60.• Klein AM, Salemink E, de Hullu E, Houtkamp EO, Papa M, van der Molen MJ. Cognitive bias modification reduces social anxiety symptoms in socially anxious adolescents with mild intellectual disabilities: a randomized controlled trial. J Autism Dev Disord. 2018;48:3116-26. https://doi.org/10.1007/s10803018-3579-9. (This is one of the few CBM-I studies that have focused on NDD. It improved threat interpretation bias in socially anxious adolescents with mild ID, as well as social anxiety at ten-week follow-up).

61. Flood AM, Hare DJ, Wallis P. An investigation into social information processing in young people with Asperger syndrome. Autism. 2011;15:601-24. https://doi.org/10.1177/1362361310 387803.

62. Hollocks MJ, Pickles A, Howlin P, Simonoff E. Dual cognitive and biological correlates of anxiety in autism spectrum disorders. J Autism Dev Disord. 2016;46:3295-307. https://doi.org/ 10.1007/s10803-016-2878-2.

63. Bryan T, Sullivan-Burstein K, Mathur S. The influence of affect on social-information processing. J Learn Disabil. 1998;31:41826. https://doi.org/10.1177/002221949803100501.

64. Mikami AY, Hinshaw SP, Lee SS, Mullin BC. Relationships between social information processing and aggression among adolescent girls with and without ADHD. J Youth Adolesc. 2008;37:761-71. https://doi.org/10.1007/s10964-007-9237-8.

65. Colalillo S, Williamson D, Johnston C. Attributions for parents' behavior by boys with and without attention-deficit/hyperactivity disorder. Child Psychiatry Hum Dev. 2014;45:765-75. https:// doi.org/10.1007/s10578-014-0445-7.

66. Andrade BF, Waschbusch DA, Doucet A, King S, MacKinnon $\mathrm{M}, \mathrm{McGrath} \mathrm{PJ}$, et al. Social information processing of positive and negative hypothetical events in children with ADHD and conduct problems and controls. J Atten Disord. 2012;16:491504. https://doi.org/10.1177/1087054711401346.

67. King S, Waschbusch DA, Pelham WE, Frankland BW, Andrade $\mathrm{BF}$, Jacques S, Corkum PV. Social information processing in elementary-school aged children with ADHD: medication effects and comparisons with typical children. J Abnorm Child Psychol. 2009;37:579-89. https://doi.org/10.1007/s10802-008-9294-9.

68. Sibley MH, Evans SW, Serpell ZN. Social cognition and interpersonal impairment in young adolescents with ADHD. J Psychopathol Behav Assess. 2010;32:193-202. https://doi.org/10. 1007/s10862-009-9152-2.

69. Haas SM, Waschbusch DA, King S, Walsh TM. Examining the role of callous-unemotional traits in the attributional styles and self competence evaluations of children with conduct problems and ADHD. J Psychopathol Behav Assess. 2015;37:196-206. https://doi.org/10.1007/s10862-014-9459-5.

70. AlMoghrabi N, Huijding J, Mayer B, Franken IH. Gaze-contingent attention bias modification training and its effect on attention, interpretations, mood, and aggressive behavior. Cogn Ther Res. 2019;58:36. https://doi.org/10.1007/s10608-019-10010-5.

71. Kaiser D, Jacob GA, van Zutphen L, Siep N, Sprenger A, Tuschen-Caffier B, et al. Biased attention to facial expressions of ambiguous emotions in borderline personality disorder: an eye-tracking study. J Pers Disord. 2019;33:671-S8. https://doi. org/10.1521/pedi_2019_33_363.

72 van Nieuwenhuijzen M, Orobio de Castro B, Wijnroks L, Vermeer A, Matthys W. The relations between intellectual disabilities, social information processing, and behaviour problems. Eur J Dev Psychol. 2004;1:215-29. https://doi.org/10.1080/17405 620444000111.

73. Ziv Y, Hadad BS, Khateeb Y, Terkel-Dawer R. Social information processing in preschool children diagnosed with autism 
spectrum disorder. J Autism Dev Disord. 2014;44:846-59. https://doi.org/10.1007/s10803-013-1935-3.

74. Mazza M, Mariano M, Peretti S, Masedu F, Pino MC, Valenti M. The role of theory of mind on social information processing in children with autism spectrum disorders: a mediation analysis. J Autism Dev Disord. 2017;47:1369-79. https://doi.org/10.1007/ s10803-017-3069-5.

75. Meyer JA, Mundy PC, van Hecke AV, Durocher JS. Social attribution processes and comorbid psychiatric symptoms in children with Asperger syndrome. Autism. 2006;10:383-402. https://doi. org/10.1177/1362361306064435.

76. Dodge KA, Godwin J. Social-information-processing patterns mediate the impact of preventive intervention on adolescent antisocial behavior. Psychol Sci. 2013;24:456-65. https://doi. org/10.1177/0956797612457394.

77. Waschbusch DA. A meta-analytic examination of comorbid hyperactive-impulsive-attention problems and conduct problems. Psychol Bull. 2002;128:118-50. https://doi.org/10.1037// 0033-2909.128.1.118.

78. Schmidt NB, Vereenooghe L. Targeting hostile attributions in inclusive schools through online cognitive bias modification: a randomised experiment. Behav Res Ther. 2021;146:103949. https://doi.org/10.1016/j.brat.2021.103949. (This RCT found CBM-I to be effective in reducing characterological otherblame in a mixed sample of children with and without NDD who attended inclusive schools).

79. Schmidt NB, Vereenooghe L. Inclusiveness of cognitive bias modification research toward children and young people with neurodevelopmental disorders: a systematic review. Int J Dev Disabil. 2020; Advanced Online Publication. https://doi.org/10. 1080/20473869.2020.1720156. (This systematic review identified 29 CBM studies targeting biased interpersonal ambiguity processing. All studies targeted interpretation bias and one third explicitly excluded CYP with NDD, cognitive deficits or special needs. The three studies that included CYP with NDD found CBM to be effective at reducing cognitive bias in CYP with mild ID, ASD or ADHD).

80. Downes MJ, Brennan ML, Williams HC, Dean RS. Development of a critical appraisal tool to assess the quality of cross-sectional studies (AXIS). BMJ Open. 2016;6: e011458. https://doi.org/10. 1136/bmjopen-2016-011458.

Publisher's Note Springer Nature remains neutral with regard to jurisdictional claims in published maps and institutional affiliations. 